\title{
Tratamiento endovascular de pseudoaneurisma roto de pseudoquiste pancreático
}

\author{
Endovascular treatment of a ruptured pseudoaneurysm of a pancreatic \\ pseudocyst
}

\author{
Javier Cóggiola', Camila C. Battú2 , Diego Juárez³, Rubén A. Retamar, Raúl Schmuck ${ }^{5}$
}

\section{RESUMEN}

Los pseudoquistes pancreáticos aparecen en el 5-40\% de las pancreatitis crónicas. Sus complicaciones vasculares no son frecuentes; estas pueden producir erosión de los vasos adyacentes por compresión, isquemia o destrucción enzimática de las paredes, que dan lugar a la formación de pseudoaneurismas de las arterias adyacentes y pueden causar hemorragia intraquística. La rotura del pseudoaneurisma constituye la complicación más grave de la pancreatitis, y es más frecuente en la crónica que en la aguda, con una presentación del 2-10\%. La angiografía es un método invasivo que permite el diagnóstico y la conducta terapéutica.

Palabras claves: pseudoquiste pancreático; pancreatitis crónica; angiografía; embolización; hemorragia digestiva alta.

\begin{abstract}
The pancreatic pseudocysts appear in 5-40\% of chronic pancreatitis, their vascular complications are not frequent but can produce erosion of the adjacent vessels by compression, ischemia or enzymatic destruction of the walls, leading to the formation of pseudoaneurysms of the adjacent arteries causing intracystic hemorrhage. The rupture of the pseudoaneurysm is the most serious complication of pancreatitis, being more frequent in the chronic than in the acute, with a presentation of 2-10\%. Angiography is an invasive method, which allows the diagnosis and therapeutic behavior.
\end{abstract}

Keywords: pancreatic pseudocyst; chronic pancreatitis; angiography; embolization.

Revista Argentina de Cardioangiología Intervencionista 2018;9(4):220-222. DOI: 10.30567/RACI/201804/0220-0222

\section{CASO CLÍNICO}

Paciente masculino de 33 años, etilista de jerarquía, monorreno por nefrectomía izquierda en 2009 por litiasis renal, con antecedente de pseudoquiste pancreático por pancreatitis enólica con drenaje endoscópico en 2016, con mala adherencia a los controles posprocedimiento.

El paciente reingresa en junio de 2017 por hemorragia digestiva alta, se realiza endoscopia digestiva donde no se evidencian signos de sangrado activo, se extrae stent doble pigtail migrado con coágulo en su extremo. Ante la persistencia de signos clínicos y bioquímicos de hemorragia activa se solicita tomografía computarizada (TC) con contraste endovenoso (EV); en ella se evidencia la presencia de formación pancreática de $100 \times 96 \mathrm{~mm}$ con extravasación de contraste en su interior, compatible con pseudoaneurisma de pseudoquiste pancreático con sangrado activo intraquístico (Figura 1).

Se solicita arteriografía de urgencia. Mediante punción femoral derecha, se realiza cateterización selectiva de arteria hepática con catéter Simmons 1 (Terumo, Japón), constatándose en control angiográfico sangrado activo hacia cavi-

1. Médico Cardiólogo. Fellow de Cardiología intervencionista. Servicio de Hemodinamia, Hospital J.M. Cullen

2. Médico Residente. $4^{\circ}$ año. Cirugía General y Mixta, Hospital J.M. Cullen

3. Médico Cirujano. Subjefe de Servicio de Cirugía General y Mixta, Hospital J.M. Cullen

4. Médico Cardiólogo Intervencionista. Jefe de Servicio de Hemodinamia, Hospital J.M. Cullen

5. Médico Cardiólogo Intervencionista. Servicio de Hemodinamia, Hospital J.M. Cullen

$\triangle$ Correspondencia: Dr. Javier Cóggiola. Av. Freyre 2150, CP 3000 Santa Fe, Santa Fe, Rep. Argentina.Email: javierco16@hotmail.com

Los autores no declaran conflictos de intereses

Recibido: 14/03/2018|Aceptado: 28/05/2018 dad (Figura 2). Se cateteriza superselectivamente arteria gastroduodenal con microcatéter Progreat (Terumo, Japón) detectándose a esta como la responsable del cuadro. Se logra avanzar catéter guía, a través del cual se libera un coil (Azur, Terumo, Japón) de manera satisfactoria. El control angiográfico posterior muestra estagnación de sustancia de contraste sin sangrado evidente (Figura 3).

El paciente presenta buena evolución posprocedimiento, pasa a sala general, donde permanece estable hemodinámicamente, requiriendo transfusión de 2 unidades de glóbulos rojos ante el descenso posembolización de hemoglobina/hematocrito. Se retira de alta hospitalaria al $5^{\circ}$ día posembolización.

En agosto de 2017 se realiza TC control donde se evidencia la remisión del pseudoquiste sin sangrado activo en cavidad (Figura 4).

\section{DISCUSIÓN}

El pseudoquiste pancreático es una complicación de la pancreatitis aguda o crónica con una incidencia del 5 al 50\% según las diferentes series.

Las complicaciones vasculares de la pancreatitis son poco frecuentes; habitualmente se presentan en pancreatitis crónica, en su mayor parte de tipo enólica. Su importancia radica en su alta tasa de morbimortalidad ${ }^{1}$.

Los pseudoquistes pancreáticos o los estados inflamatorios crónicos en la pancreatitis pueden producir erosión de los vasos adyacentes por diferentes mecanismos: compresión persistente, isquemia o destrucción de la pared vascular por la acción de sus enzimas. Esto puede dar lugar a hemorragia intraquística, hemorragia intra- o retroperitoneal, trombosis venosa o a la formación de pseudoaneurismas en las arterias adyacentes ${ }^{1}$. 


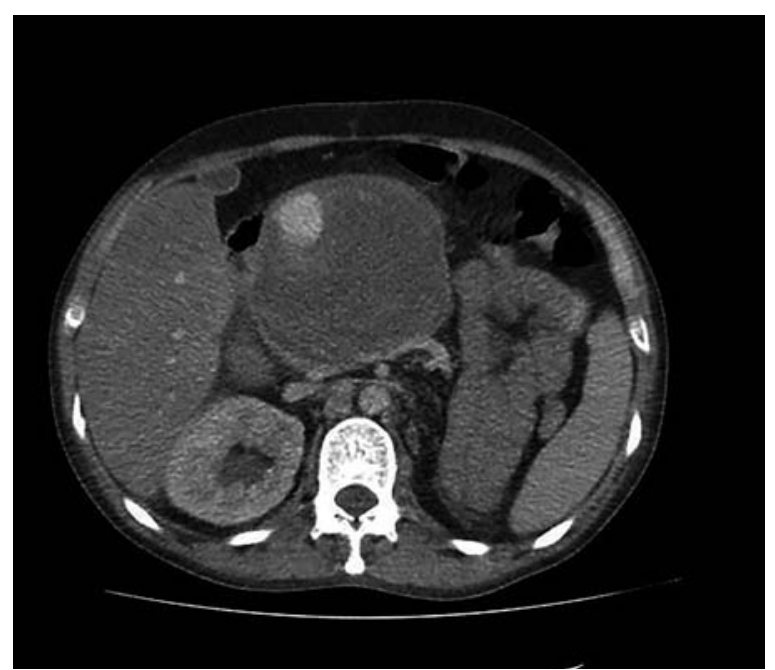

Figura 1. Imagen topográfica que evidencia formación quística pancreática de 100×96 mm, con extravasación de contraste activa en fase arterial y contenido heterogéneo en su interior.

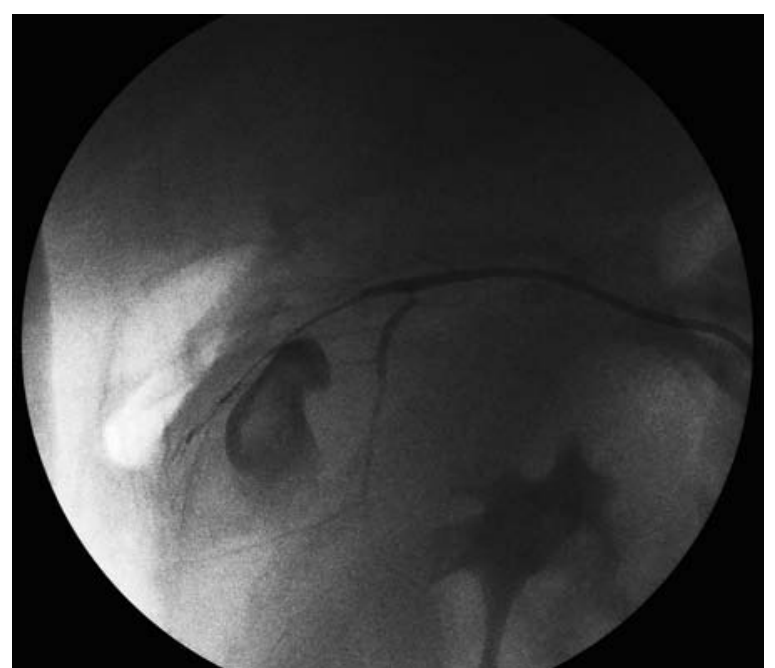

Figura 2. Imagen angiográfica compatible con pseudoaneurisma a nivel de la arteria gastroduodenal.

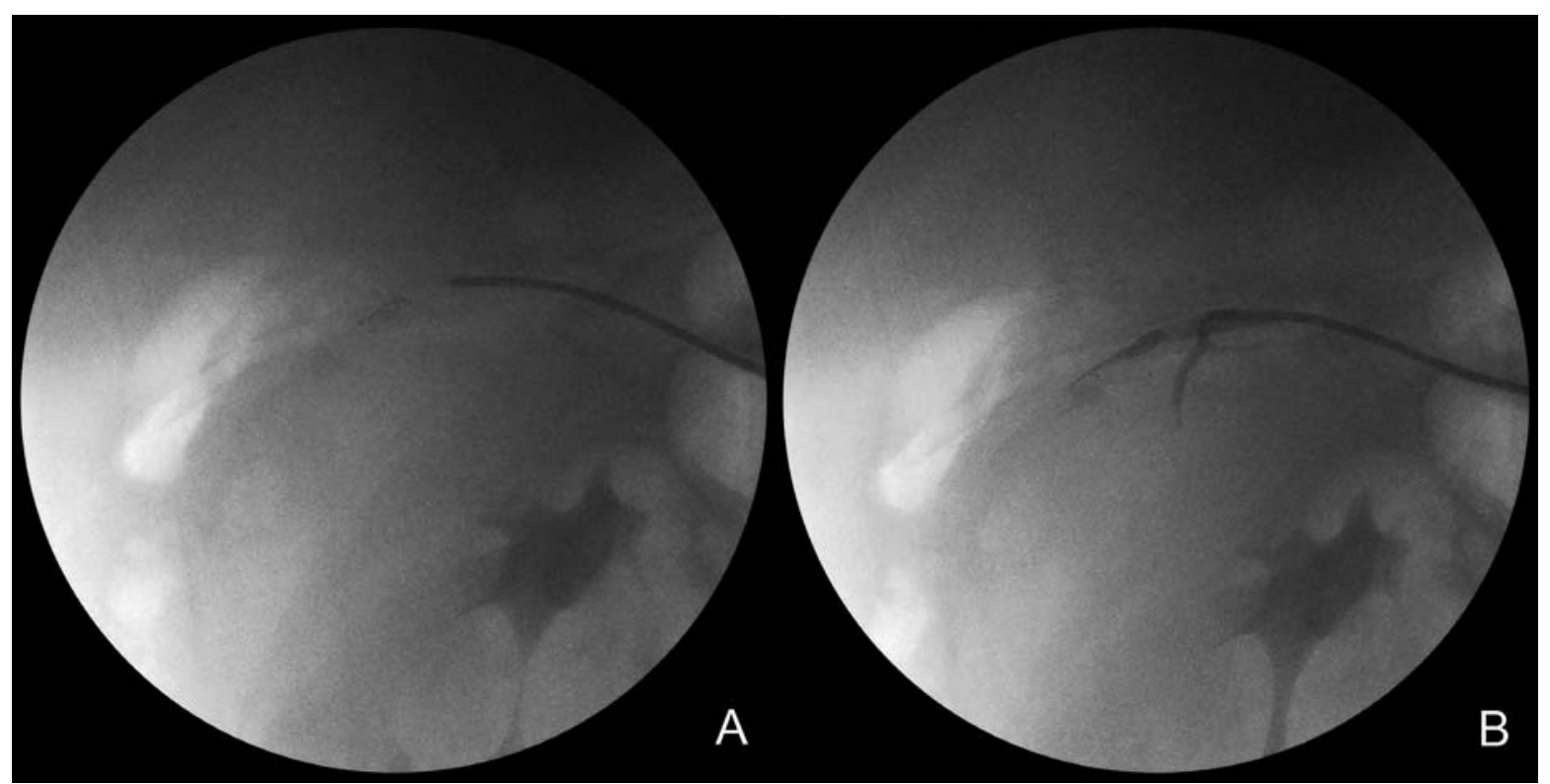

Figura 3. Control angiográfico posterior a liberación de coil. A. Coil posicionado en segmento proximal de arteria gastroduodenal. B. Estagnación de contraste, con desaparición de pseudoaneurisma.

En un $10 \%$ de los pacientes con pseudoquistes se ha descrito la formación de pseudoaneurismas. Las arterias más afectadas son la esplénica, la gastroduodenal, las pancreático-duodenales y la hepática. La rotura del pseudoaneurisma es la complicación más grave de la pancreatitis, con una prevalencia de hasta el $10 \%$ y mortalidad cercana al $90 \%^{2}$.

Solo un $2,5 \%$ de estas complicaciones se presentan clínicamente con hemorragia digestiva, la cual se debe a la rotura del pseudoaneurisma o la erosión del pseudoquiste sobre la pared del aparato digestivo. Las hemorragias digestivas altas son aquellas que tienen origen por encima del ligamento de Treitz, y son las responsables de hasta el 76\% de los eventos hemorrágicos gastrointestinales. La localización del sangrado, previo al ingreso a la Sala de Hemodinamia, es de capital importancia para un abordaje más dirigido. Por esto la endoscopia debería ser la intervención inicial ya que permite identificar, en la mayoría de los casos, la localización, descartar etiologías más frecuentes (úlcera péptica sangrante, várices esofágicas, síndrome de Mallory-Weis) y una eventual terapéutica.
Ante pacientes con endoscopia digestiva negativa se debe avanzar en exámenes complementarios. La tomografía computarizada contrastada ha demostrado ser capaz de evidenciar sangrados de hasta $0,3 \mathrm{ml} / \mathrm{min}$, mejor que la angiografía convencional, con lo cual debería estar disponible previo al procedimiento invasivo. Además puede ser útil para diagnosticar variantes anatómicas y ángulo de nacimiento de los vasos mesentéricos y así planificar el procedimiento con antelación. También, aunque generalmente menos disponible y de poca utilidad en el episodio agudo, puede utilizarse la medicina nuclear con glóbulos rojos marcados con Tc-99 m. La angiografía puede detectar sangrados con tasas de hasta $0,5 \mathrm{ml} / \mathrm{min}$. Los hallazgos angiográficos sugestivos de sangrado son: la extravasación activa de contraste, la retención de contraste durante la fase venosa como signos directos, y la visualización de vasoespasmo o interrupción abrupta del vaso, la presencia de pseudoaneurismas, la hipervascularización del lecho o retorno venoso precoz como signos indirectos. 


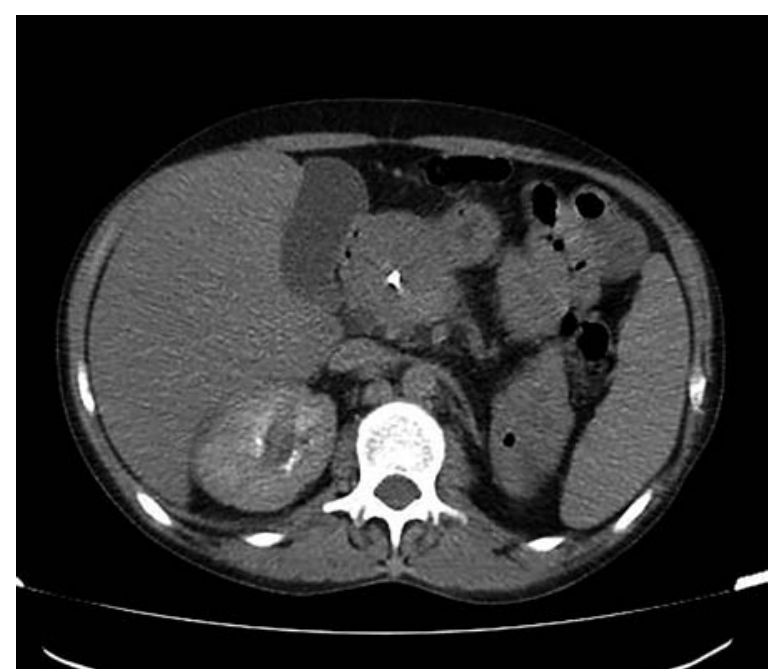

Figura 4. Control tomográfico al primer mes posembolización. Remisión del pseudoquiste pancreático sin extravasación de contraste. Flecha: coil.

En caso de que no se constate sangrado activo en la angiografía, se debería proceder a la cateterización superselectiva con microcatéter del vaso secundario sospechado de ser el causante ${ }^{3}$.

Loffroy et al. reportaron una tasa de éxito técnico y clínico de $93 \%$ y $67 \%$ con un $33 \%$ de resangrados, en los cuales una nueva embolización demostró ser efectiva en la mayoría de los casos ${ }^{4}$.

En pacientes hemodinámicamente inestables, o en aquellos con alta sospecha diagnóstica, la arteriografía abdo-

\section{BIBLIOGRAFÍA}

1. DePrado N, Corral de la Calle M, Gallardo Sánchez F, et al. Complicaciones vasculares de la pancreatitis. Rev Clin Esp 2005;205(7): 326-32.

2. Larrey-RuizL, Luján-SanchisM, Peño-MuñozL, etal. Pseudoaneurismaasociado apseudoquiste pancreático complicado. RevEsp Enferm Dig 2016;108(9):583-5.

3. Sierre S. Tratamiento endovascular de la hemorragia digestiva. Embolización digestiva. Revista Argentina de Cardioangiología 2011;(2):0049-0053. minal es una alternativa segura con fines diagnósticos y terapéuticos. Una vez localizado el sitio del sangrado se procede a la embolización de forma selectiva mediante la introducción de coils en el aneurisma sangrante o en su arteria nativa.

La cirugía debería reservarse para los pacientes hemodinámicamente inestables sin respuesta a la reanimación inicial, en pacientes en los que no se logra definir o cateterizar selectivamente el sitio de sangrado para su embolización o en aquellos en los que haya fracasado el intento de embolización ${ }^{5}$.

\section{CONCLUSIONES}

Las complicaciones vasculares de las pancreatitis crónicas son poco frecuentes pero tienen una mortalidad elevada, con lo cual el diagnóstico y tratamiento oportuno cobra capital importancia. El creciente desarrollo de las terapéuticas endovasculares permite el tratamiento efectivo en un gran porcentaje de estos pacientes, lo cual resulta particularmente oportuno debido a las comorbilidades que suelen presentar, las que los convierten en malos candidatos quirúrgicos. Es imprescindible la creación de un equipo multidisciplinario y un algoritmo de actuación ante estos cuadros, de acuerdo a la disponibilidad de cada centro.

\section{AGRADECIMIENTOS}

Se agradece la participación y colaboración durante el procedimiento al técnico radiólogo Alejandro Rosa.
4. Loffroy $R$, et al. Embolization of acute nonvariceal upper gastrointestinal hemorrhage resistant to endoscopic treatment: results and predictors of recurrent bleeding. Cardiovasc Intervent Radiol 2010;33:1088-1100.

5. Guimaraes, et al. Embolization therapy: principles and clinical applications. Ed. Wolsters Kluwer. 2015. 